\title{
CHANGES IN THE HEMOSTATIC SYSTEM AGAINST THE BACKGROUND OF VARIOUS TREATMENT REGIMENS IN PATIENTS WITH ATHEROSCLEROSIS OF LOWER EXTREMITIES
}

\author{
Ivan Tverdovsky ${ }^{l}$ \\ Ivan Tutrin \\ Department of Anesthesiology and Resuscitation \\ Siberian State Medical University \\ 2 Moskovsky trakt, Tomsk, Russia, 634055 \\ Oleg Tarabrin ${ }^{1}$ \\ ${ }^{1}$ Department of Anesthesiology, Intensive Care with Postgraduate Training \\ Odessa National Medical University \\ 2 Valikhovsky lane, Odessa, Ukraine, 65028
}

\begin{abstract}
About 2-3\% of the population and about 35-50\% of people over 65 years suffer from obliterating atherosclerosis of the main arteries of the lower extremities. There are many known factors that potentiate atherosclerosis. Some of them are referred to as leading, such as hyperlipoproteinemia or hypertension, others to concomitant contributing to the defeat of the arteries. According to J.S.A. Fuchs include hypertension, hypercholesterolemia and smoking as major risk factors for atherosclerosis. They are less affected by obesity, diabetes, hypertriglyceridemia, sedentary lifestyle, stress

Objective of the study. The purpose of this study is to evaluate the functional status of the vascular - platelet, coagulation and fibrinolysis of the hemostasis system and their disorders using low - frequency piezoelectric thromboelastography (LPTEG) in patients with obliterating atherosclerosis of the lower extremities on the background of treatment.

Materials and methods. The initial state of the hemostasis system was evaluated in 90 patients undergoing treatment for obliterating atherosclerosis of the lower extremities in the Odessa Regional Clinical Hospital and MD Odreks. All patients underwent surgery in the amount of femoral-popliteal autologous bypass grafting. Patients in the control (1st) group (45 patients) received analgesia during surgery and in the postoperative period based on epidural administration of bupivacaine, and their treatment regimen included enoxaparin $0.4 \mathrm{ml}$ (40,000 anti-XA ME) 2 times a day subcutaneously into the anterolateral surface of the abdominal wall from the first postoperative day and pentoxifylline IV $200 \mathrm{mg}$ twice a day for 7 days. Patients of the studied (2nd) group (45 patients) received analgesia during surgery and in the postoperative period on the basis of epidural administration of bupivacaine, also rivaroxaban $15 \mathrm{mg}$ 2 times a day oraly on the first postoperative day was included in their treatment regimen.

The dynamics of the state of the hemostatic system in both groups of patients with OA was evaluated using LPTEG in the first day before surgery and on the 3 rd, 5 th and 7 th postoperative days.

Results and conclusions. Before surgery and the start of treatment in patients with OA, against the background of activation of the vascular - platelet link of hemostasis, significant structural and chronometric hypercoagulation with increased thrombin generation and activation of blood fibrinolytic activity are noted. Against the background of treatment in the 1st group, platelet aggregation tends to normalize and there are significant differences compared with the indicators before treatment in the 1st group, the vascular-platelet and fibrinolytic systems did not reach normal values before 7 days, but showed a significant downward trend the degree of manifestation of their violations. Against the background of treatment of the 2nd group, significant changes were observed towards normalization in all components of the hemostatic system according to the LPTEG technique. The state of the coagulation unit of the hemostasis system in patients with OA on the 7th day of therapy does not significantly differ from the norm, it is possible to note significant shifts towards hypocoagulation within the normal range. The fibrinolytic system on the 7th day of treatment does not statistically differ from normal indicators, it has significant positive dynamics compared to 1 day. The second group revealed a statistically significant normalization of all indicators characterizing the blood coagulation potential of the blood and the aggregation ability of platelets, as well as the indicator that is responsible for changes in the fibrinolytic blood system.
\end{abstract}

Keywords: obliterating atherosclerosis, thrombus formation, hemostasis. 


\section{Introduction}

About $2-3 \%$ of the population and about $35-50 \%$ of people over 65 years suffer from obliterating atherosclerosis of the main arteries of the lower extremities $[1,2]$. The problem of treatment of obliterative atherosclerosis $(\mathrm{OA})$ of the vessels of the lower extremities is currently very relevant [3]. There are many known factors that potentiate atherosclerosis [4]. Some of them are referred to as leading, such as hyperlipoproteinemia or hypertension, others to concomitant contributing to the defeat of the arteries $[5,6]$.

In addition, many researchers emphasize the poly factor of the process of atherogenesis, including the adverse effect of hemodynamic factors on the arterial wall, vasoactive substances, endo- and exogenous chemical compounds, dyslipoproteinemia of atherogenic nature, other disorders $[7,8]$. Special attention is paid to the role of early metabolic disorders of the arterial wall structure and the importance of lipoprotein oxidation in it $[9,10]$.

The association of an increased level of a number of factors of the hemostasis system with the development of atherosclerosis and its complications such as stroke and myocardial infarction has been demonstrated in a number of epidemiological studies [11, 12]. In hyperlipidemia, in addition to the increase in platelet activity, there is a decrease in antithrombogenic (antiaggregation, anticoagulant, fibrinolytic) activity of the vessel wall, increase in factor VII, fibrinogen, Villebrand factor and plasminogen activator inhibitor $[13,14]$. According to J.S.A. Fuchs include hypertension, hypercholesterolemia and smoking as major risk factors for atherosclerosis [15]. They are less affected by obesity, diabetes, hypertriglyceridemia, sedentary lifestyle, stress [16]. Increased mortality is mainly driven by a higher cardiovascular disease risk and reflects the underlying systemic atherosclerosis process in peripheral arterial disease patients [17]

Increase in the concentration of Willibrand factor in the blood is a marker of endothelial damage and dysfunction, its increase is associated with the risk of vascular complications in patients with disorders of lipid metabolism $[18,19]$. Damage to the atherosclerotic plaque is a major link in further thrombogenesis. The lipid-rich plaque contains a tissue factor that locally initiates activation of the coagulation system $[20,21]$. It is widely acknowledged that anticoagulant medication therapy is an integral part of the prevention of cardiovascular mortality and morbidity in this category of patients [22].

The aim of the study. The purpose of this study is to evaluate the functional state of the vascular - platelet, coagulation unit and fibrinolysis of the hemostasis system and their disorders by means of low - frequency piezoelectric thromboelastography (LPTEG) in patients with obliterating atherosclerosis of the lower extremities.

\section{Materials and methods}

Clinical material for the study is presented by 90 patients who have undergone treatment for obliterating atherosclerosis (OA) in the period from 2017 to 2019 on the basis of the departments of surgery and intensive care of the Odessa Regional Clinical Hospital. The criteria for inclusion in the study were: the presence of a patient with obliterating atherosclerosis (OA) IIb and III stage, verified by computer angiography or Doppler ultrasound, stenosis of the femoral, popliteal or tibial segment, age 18 years or older, the absence of factors indicated exclusion criteria, informed written consent of the patient to participate in the study. Exclusion criteria for the study were: refusal of the patient to participate in the study, refusal of patients from the components of therapy: transfusion of blood components (for religious reasons), epidural anesthesia and analgesia (fear of complications of puncture of the epidural space), the presence of relative or absolute opposite the presence of allergic reactions to lidocaine or bupivacaine, uncompensated chronic diseases or diseases that affect the hemostatic system.

The study is in compliance with the legislation of Ukraine on medical ethics and was approved at the meeting of the Commission on Bioethics of the Odessa National Medical University (protocol No. 147 B of May 21, 2017).

All patients provided informed written consent to participate in the study.

Evaluation of the hemostasis system was carried out using LPTEG. Blood sampling for the study was performed under the same conditions according to the standard method from cubital vein 
of the subjects. To study the system of hemostasis and rheological properties of blood, hardware and software complex ARP-01M «Mednord» was used for continuous registration of the basic parameters of the process of blood clot formation and its lysis.

All patients underwent surgical intervention in the volume of the hip-hamstring bypass with an auto vein. Adding a patient with OA to a particular group occurred randomly. Patients of both groups were examined in the same way, received the same intensive care regimen, indications for surgical intervention were set on the basis of the same criteria. Patients in the control (1st) group (45 patients) received analgesia during surgery and in the postoperative period based on epidural administration of bupivacaine, and their treatment regimen included enoxaparin $0.4 \mathrm{ml}$ (40 000 anti-XA ME) 2 times a day subcutaneously into the anterolateral surface of the abdominal wall from the first postoperative day and pentoxifylline into vein $200 \mathrm{mg}$ twice a day for 7 days. Patients of the studied (2nd) group (45 patients) received analgesia during surgery and in the postoperative period on the basis of epidural administration of bupivacaine, also rivaroxaban $15 \mathrm{mg} 2$ times a day oraly on the first postoperative day and pentoxifylline into vein $200 \mathrm{mg}$ twice a day for 7 days was included in their treatment regimen. The group of healthy volunteers consisted of 45 people, aged 45 to 65 years, undergoing a planned annual medical examination and not having any pathology.

The dynamics of the state of the hemostatic system in both groups of patients with OA was evaluated using LPTEG in the first day before surgery and on the 3rd, 5th and 7th postoperative days. OA treatment included gastroprotective, rheologically, antioxidant, metabolic and infusion therapy.

Statistical processing of the obtained results was performed using the formulas known in medical statistics and consisted of determination of average values of numerical characteristics of the studied process, numerical characteristics of the degree of process variability, estimation of confidence interval and significance level, correlation analysis and determination of correlation reliability. Most of the numerical data obtained from the study were subjected to statistical analysis using the Student's t test with the calculation of arithmetic mean (M), root mean square deviation $(\delta)$, criterion for reliability of difference of mean values $(\mathrm{t})$. Differences were considered significant if $\mathrm{p}$ is less than 0.05 , which corresponds to $95 \%$ and is more likely to make an error. The results of the study were mathematically processed after the database was created on the Microsoft system using a suite of programs integrated into the Microsoft Office system using the Student's t test.

\section{Results}

In patients with OA, a significant structural (increase in the amplitude of the maximum density of the clot (MA LPTEG)) and chronometric (reduction of the intensity of the contact phase of coagulation (ICC), the intensity of coagulation drive (the intensity of clot formation (ICD), a constant thrombin activity (CTA) and blood clotting time (BCT(t3)) of LPTEG) hypercoagulation with increased thrombin generation (initial rate of aggregation of blood at the time $\mathrm{t} 0$ (A0), the time the contact phase of coagulation (R(t1)), the clot intensity of the polymerization (CIP LPTEG)) and depression of fibrinolysis - the intensity of the retraction and clot lysis (IRCL).

After surgery according to LPTEG (Table 1) in the first group, changes in indicators toward hypercoagulation were established: in patients with OA, numerous structural (increase in MA) and chronometric (reduction of ICC, CTA and ICD, BCT reduction were noted in patients with OA, hypercoagulation with increased thrombin activity (A0, R (t1), ICC) and depression of fibrinolysis (IRCL).

The recorded amplitude of the indicator of the intensity of the contact phase coagulation (ICC) $145,41 \pm 3,23$ r.u. (in the 1st group), at the rate of $84.3 \pm 10.91$ r.u., the initial indicator of the aggregate state of blood at the time of $\mathrm{AO} 435,49 \pm 26,88$ r.u. (in the 1st group), at the rate of $222.25 \pm 15.33$ r.u., the coagulation contact phase time $\mathrm{R}$ (t1) is $1.36 \pm 0.34$ minutes (in the 1st group) at a rate of $2.36 \pm 0.34$, all of which indicates an increase in spontaneous platelet aggregation. An increase in the amplitude and shortening of the time constants of hemocoagulation were also noted. When comparing the results with the healthy group, the following data were obtained: an increase in the constant thrombin activity (CTA) by $104 \%$ in the first group, an increase in the blood clotting time (BCT (t3)) by $47.26 \%$ in the first group, an increase in the intensity of coagulation drive 
(ICD) by $97.3 \%$ in the first group, an increase in maximal density of the clot (MA) by $74.03 \%$ in the first group, an decrease in the rate of retraction and clot lysis (IRCL) by $47.29 \%$ in the first. In the second group, similar changes were observed before the start of treatment compared to the group of healthy volunteers. Thus, the A0 increased by $88.81 \%$, the R ( $\mathrm{t} 1$ ) increased by $40.38 \%$, the CTA increased by $97.89 \%$ compared to the norm. The MA indicator in the second group increased by $57.16 \%$ compared to the indicator of healthy volunteers $(825.81 \pm 51.87$ r.u. vs. $525.45 \pm 60.50)$. In the second group, the activation of the fibrinolysis system was observed, an decrease in IRCL by $39.33 \%$ compared to the norm.

\section{Table 1}

The state of hemostasis in patients with OA before treatment $(\mathrm{M} \pm \mathrm{m})$

\begin{tabular}{ccccc}
\hline No. & Indicator & Healthy group & 1(st) group & 2(nd) group \\
\hline 1 & $\mathrm{~A} 0$ & $222.25 \pm 15.33$ & $435.49 \pm 26.88^{*}$ & $419.65 \pm 23.12^{*}$ \\
2 & $\mathrm{R}\left(\mathrm{t}_{1}\right)$ & $2.36 \pm 0.34$ & $1.36 \pm 0.34^{*}$ & $1.48 \pm 0.11^{*}$ \\
3 & $\mathrm{ICC}$ & $84.3 \pm 10.91$ & $145.41 \pm 3.23^{*}$ & $139.74 \pm 3.03^{*}$ \\
4 & $\mathrm{CTA}$ & $15.22 \pm 3.46$ & $31.13 \pm 0.54^{*}$ & $30.12 \pm 0.51^{*}$ \\
5 & $\mathrm{BCT}\left(\mathrm{t}_{3}\right)$ & $8.42 \pm 1.68$ & $4.44 \pm 0.39^{*}$ & $5.02 \pm 0.29^{*}$ \\
6 & $\mathrm{ICD}$ & $21.15 \pm 3.70$ & $41.73 \pm 1.29^{*}$ & $37.59 \pm 0.93^{*}$ \\
7 & $\mathrm{CIP}$ & $14.45 \pm 1.4$ & $31.47 \pm 0.87^{*}$ & $28.43 \pm 0.25^{*}$ \\
8 & $\mathrm{MA}$ & $525.45 \pm 60.50$ & $914.49 \pm 61.13^{*}$ & $825.81 \pm 51.87^{*}$
\end{tabular}

Note: differences are significant in comparison with the healthy group, ${ }^{*}-p<0.05$

On the third day after surgery in both groups, we observed statistically significant $(\mathrm{p}<0.05)$ changes towards hypercoagulation with moderate dynamics toward normalization. Thus, the preservation of activation of the platelet component of hemostasis was indicated by an increase in the degree of A0 by $88.81 \%$ in the 1 st group and $46.42 \%$ in the 2 nd group; R (t1) was $37.28 \%$ in group 1 and $10.16 \%$ in group 2, and there was also a change in ICC of $65,76 \%$ in group 1 and $18.23 \%$ in group 2 in compared to healthy volunteers.

A statistically significant deviation from the norm of LTPEG indices was revealed (Table 2), namely A0, R (t1) and ICC, which characterize platelet aggregation capacity. Thus, the registered amplitude of the indicator of the intensity of the contact phase of coagulation (ICC) $139.74 \pm 3.03$ r.u. in group 1 and $119.23 \pm 1.78$ r.u. in group 2, at a rate of $84.3 \pm 10.91$ r.u., the initial indicator of the aggregate state of blood at time A0 $419.65 \pm 23,12$ r.u. in 1 group and $325.44 \pm 22.34$ r.u. in group 2, at the rate of $222.25 \pm 15.33$ r.u., the contact phase of coagulation time $(\mathrm{R}(\mathrm{t} 1))$ is $1.48 \pm 0.11$ minutes in group 1 and $2.03 \pm 0.18$ minutes in group 2 , with a rate of $2.36 \pm 0.34$, all of which indicates a significant increase spontaneous platelet aggregation. An increase in the amplitude and shortening of the time constants of hemocoagulation were also noted. When comparing the results with the healthy group, the following data were obtained: an increase in the constant of thrombin activity (CTA) by $97.89 \%$ in the 1st group and by $80.74 \%$ in the 2nd group, a decrease in the blood clotting time (T3) by $40,38 \%$ in Group 1 and $21.73 \%$ in Group 2, the intensity of coagulation drive (ICD) accelerated by $77.73 \%$ in Group 1 and $54.04 \%$ in Group 2, increasing the maximum density of the clot was $57.16 \%$ in Group 1 and $33.1 \%$ in Group 2. The nature of the changes indicates the presence of hypercoagulation due to increased activity and vascular-platelet and procoagulant hemostasis, as well as deviation from the norm, which characterizes fibrinolytic activity (IRCL) decrease by $39.33 \%$ in the 1 st group and by $33.09 \%$ in $2^{\text {nd }}$ group compared to healthy volunteers.

These changes in the hemostasis system indicate a decrease in hypercoagulation, hyperaggregation with the orientation of both groups toward normocoagulation, which are more significant in the second group, which we believe is associated with the use of rivaroxaban as a component of treatment in patients of the second group.

Fig. 1 shows the indicators of LPTEG on day 3 in both groups and in healthy volunteers. 
Table 2

State of hemostasis system in patients with OA for 3 days after surgery $(\mathrm{M} \pm \mathrm{m})$

\begin{tabular}{ccccc}
\hline No. & Indicator & Healthy group & 1(st) group & 2(nd) group \\
\hline 1 & A0 & $222.25 \pm 15.33$ & $419.65 \pm 23.12^{*}$ & $325.44 \pm 22.34^{*}$ \\
2 & $\mathrm{R}\left(\mathrm{t}_{1}\right)$ & $2.36 \pm 0.34$ & $1.48 \pm 0.11^{*}$ & $2.03 \pm 0.18^{*}$ \\
3 & $\mathrm{ICC}$ & $84.3 \pm 10.91$ & $139.74 \pm 3.03^{*}$ & $119.23 \pm 1.78^{*}$ \\
4 & $\mathrm{CTA}$ & $15.22 \pm 3.46$ & $30.12 \pm 0.51^{* \times}$ & $27.51 \pm 0.53^{*}$ \\
5 & $\mathrm{BCT}\left(\mathrm{t}_{3}\right)$ & $8.42 \pm 1.68$ & $5.02 \pm 0.29^{*}$ & $6.59 \pm 0.31^{*}$ \\
6 & $\mathrm{ICD}$ & $21.15 \pm 3.70$ & $37.59 \pm 0.93^{* *}$ & $32.58 \pm 1.21^{*}$ \\
7 & $\mathrm{CIP}$ & $14.45 \pm 1.4$ & $28.43 \pm 0.25^{* \times}$ & $22.81 \pm 0.49^{*}$ \\
8 & $\mathrm{MA}$ & $525.45 \pm 60.50$ & $825.81 \pm 51.87^{*}$ & $699.67 \pm 39.58^{*}$
\end{tabular}

Note: the differences are significant compared to the healthy group, ${ }^{*}-p<0.05$.

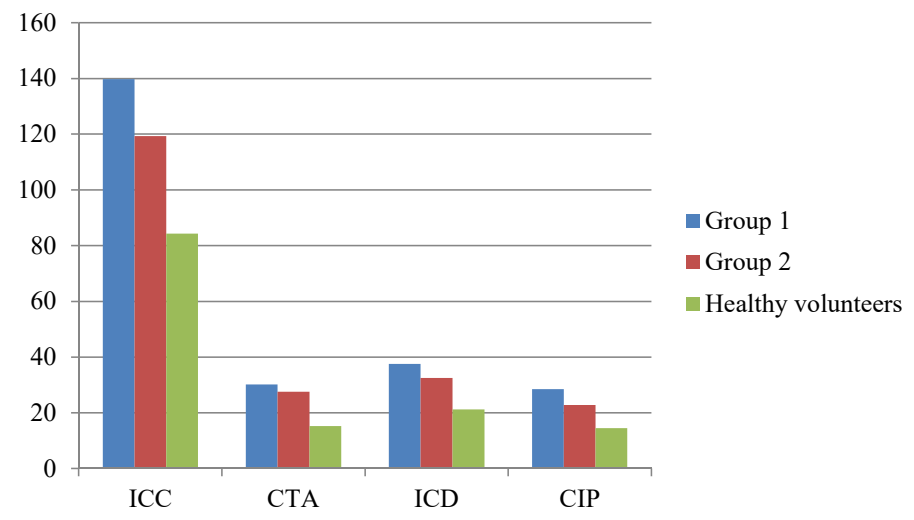

Fig. 1. Idicators of LPTEG on day 3 in both groups and in healthy volunteers

On day 5, according to the LPTEG (Table 3), the first group revealed statistically unreliable deviations from the indicators of healthy volunteers: indicators A0, R (t1) and ICC, which characterize the aggregation capacity of platelets, and the indicators that characterize hemocoagulation potential CTA, ICD, CIP, BCT as well as the indicator responsible for changes in the fibrinolytic blood system (IRCL). In the second group, these indicators are almost indistinguishable from those of the norm, indicating a more substantial change towards normalization in patients of the second group.

Table 3

Condition of hemostasis system in patients with OA for 5 days after surgery $(\mathrm{M} \pm \mathrm{m})$

\begin{tabular}{ccccc}
\hline No. & Indicator & Healthy group & 1(st) group & 2(nd) group \\
\hline 1 & $\mathrm{~A} 0$ & $222.25 \pm 15.33$ & $402.51 \pm 19.51^{*}$ & $259.49 \pm 19.87^{*}$ \\
2 & $\mathrm{R}\left(\mathrm{t}_{1}\right)$ & $2.36 \pm 0.34$ & $2.02 \pm 0.17^{*}$ & $2.12 \pm 0.18^{*}$ \\
3 & $\mathrm{ICC}$ & $84.3 \pm 10.91$ & $127.84 \pm 1.96^{*}$ & $99.67 \pm 2.02^{*}$ \\
4 & $\mathrm{CTA}$ & $15.22 \pm 3.46$ & $26.49 \pm 1.36^{*}$ & $16.31 \pm 0.50^{*}$ \\
5 & $\mathrm{BCT}\left(\mathrm{t}_{3}\right)$ & $6.42 \pm 1.68$ & $6.04 \pm 0.43^{*}$ & $8.08 \pm 0.23^{*}$ \\
6 & $\mathrm{ICD}$ & $21.15 \pm 3.70$ & $32.44 \pm 0.84^{*}$ & $22.41 \pm 1.02^{*}$ \\
7 & $\mathrm{CIP}$ & $14.45 \pm 1.4$ & $21.54 \pm 0.48^{*}$ & $15.45 \pm 0.51^{*}$ \\
8 & $\mathrm{MA}$ & $525.45 \pm 60.50$ & $759.47 \pm 39.34^{*}$ & $576.48 \pm 22.71^{*}$ \\
9 & $\mathrm{IRCL}$ & $16.45 \pm 1.40$ & $12.22 \pm 0.56^{*}$ & $15.44 \pm 0.37^{*}$
\end{tabular}

Note: differences are significant in comparison with the healthy group, ${ }^{*}-p<0.05$ 
The registered value of the intensity of the contact phase of coagulation (ICC) $127.84 \pm$ \pm 1.96 r.u. (in the 1 st group), at the rate of $84.3 \pm 10.91$ r.u., initial rate of aggregation of blood at the time t0 $402.51 \pm 19.51$ r.u. (in the 1 st group), at the rate of $222.25 \pm 15.33$ r.u., the contact phase coagulation phase R (t1) $2.02 \pm 0.17$ minutes (in the 1st group) at the rate of $2.36 \pm 0.34$, all this indicates the preservation of hyperagregation against the background of the trend towards normalization in patients the first group. Changes in the amplitude and time constants of hemocoagulation were also observed. When comparing the results with the healthy group, the following data were obtained: constant thrombin activity (CTA) was greater by $74.04 \%$ in the first group, decrease in blood clotting time (T3) changed by $28.02 \%$ in the first group, the intensity of coagulation drive ICD by $53.38 \%$ in the first group, maximum density of the clot of MA by $44.53 \%$ in the first group, intensity of retraction and clot lysis (IRCL) by $25.71 \%$ in the first.

In the second group statistically significant changes towards normalization of indicators with preservation of moderate hypercoagulation were revealed: indicators characterizing hemocoagulation potential of blood CTA, BCT, ICD, CIP, MA and indicators A0, R (t1) and ICC, which characterize the aggregation capacity of platelets as well as the indicator responsible for changes in the fibrinolytic blood system. The recorded amplitude of the indicator of the intensity of the contact phase of coagulation (ICC) $99.67 \pm 2.02$ r.u. (in the 2 nd group), at the rate of $84.3 \pm 10.91$ r.u., the initial indicator of the aggregate state of blood at the time of AO 259.49 \pm 19.87 r.u. (in the 2nd group), at the rate of $222.25 \pm 15.33$ r.u., the contact phase coagulation $\mathrm{R}$ (t1) $2.12 \pm 0.18$ minutes (in the 2 nd group) at the rate of $2.36 \pm 0.34$, all this indicates a significant $(\mathrm{p}<0.05)$ increase in spontaneous platelet aggregation. Also observed is the increase in the amplitude and the reduction of the time constants of hemocoagulation. When comparing the results with the healthy group, the following data were obtained: an increase in CT by $7.16 \%$ in the second group of patients, an increase in the clotting time of CSF blood (t3) by $4.03 \%$, an increase in ICD by $5.95 \%$ and an increase in MA by $9.71 \%$. The above changes indicate that patients in the second group show a marked tendency toward normalization of all parameters of the hemostasis system, while maintaining a slight hypo-coagulation, hyperaggregation and depression of fibrinolysis.

Fig. 2 shows the indicators of LPTEG on day 5 in both groups and in healthy volunteers.

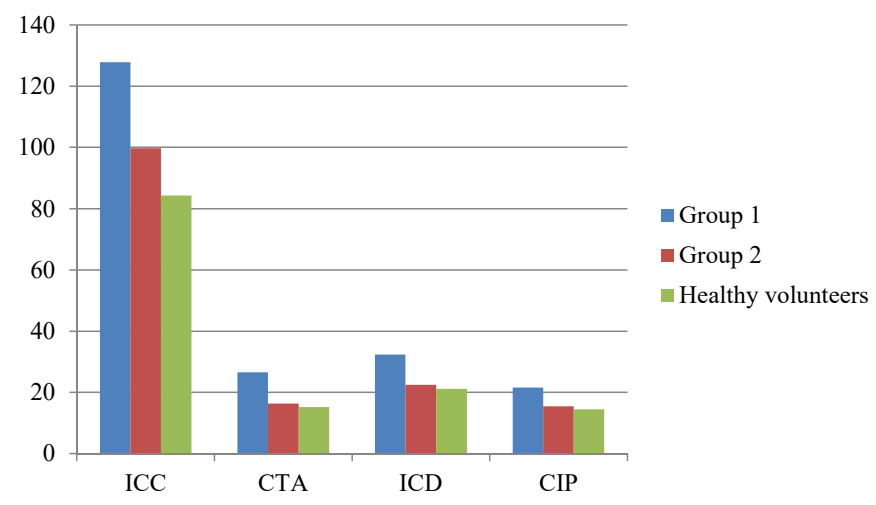

Fig. 2. Indicators of LPTEG on day 5 in both groups and in healthy volunteers

On day 7, according to the LTPEG (Table 4), in the first group, statistically significant deviation from the norm of indicators towards moderate hypercoagulation was revealed: indicators $\mathrm{A} 0, \mathrm{R}(\mathrm{t} 1)$ and ICC, which characterize the aggregation capacity of platelets and the indicators that characterize hemocoagulation, ICD, CIP, MA well as the indicator responsible for changes in the fibrinolytic blood system. In patients of the second group normalization of all indicators of the hemostasis system is noted.

The recorded amplitude of the coagulation contact phase (ICC) intensity index is $114.74 \pm$ \pm 1.78 r.u. (in the 1 st group), at the rate of $84.3 \pm 10.91$ r.u., the initial indicator of the aggregate state of blood at A0 $365.46 \pm 19.65$ r.u. (in the 1st group), at the rate of $222.25 \pm 15.33$ r.u., the contact phase of coagulation $\mathrm{R}(\mathrm{t} 1)$ is $2.19 \pm 0.08$ minutes (in the 1 st group) at the rate of $2.36 \pm 0.34$, all of which 
indicates the preservation of activation of spontaneous platelet aggregation. Consideration was also given to increasing the amplitude and reducing the time constants of hemocoagulation in patients of the first group compared with the group of healthy volunteers. When comparing the results with the healthy group, the following data were obtained: an increase in constant thrombin activity (CTA) by $9.59 \%$ in the first group, a reduction in blood clotting time (t3) by $4.51 \%$ in the first group, an increase in the intensity of coagulation drive ICD by $3.26 \%$ in the first group, increase in maximal density of the clot MA by $15.2 \%$ in the first group, depression of the intensity of the retraction and clot lysis (IRCL) by $20.18 \%$ in the first.

Table 4

The state of hemostasis in patients with OA for 7 days after surgery $(\mathrm{M} \pm \mathrm{m})$

\begin{tabular}{ccccc}
\hline No. & Indicator & Healthy group & 1(st) group & 2(nd) group \\
\hline 1 & $\mathrm{~A} 0$ & $222.25 \pm 15.33$ & $365.46 \pm 19.65^{*}$ & $220.43 \pm 15.61^{*}$ \\
2 & $\mathrm{R}\left(\mathrm{t}_{1}\right)$ & $2.36 \pm 0.34$ & $2.19 \pm 0.08^{*}$ & $2.37 \pm 0.15^{*}$ \\
3 & $\mathrm{ICC}$ & $84.3 \pm 10.91$ & $114.74 \pm 1.78^{*}$ & $84.41 \pm 1.61^{*}$ \\
4 & $\mathrm{CTA}$ & $15.22 \pm 3.46$ & $16.68 \pm 0.41^{*}$ & $15.16 \pm 0.56^{*}$ \\
5 & $\mathrm{BCT}\left(\mathrm{t}_{3}\right)$ & $8.42 \pm 1.68$ & $8.04 \pm 0.31^{*}$ & $8.49 \pm 0.18^{*}$ \\
6 & $\mathrm{ICD}$ & $21.15 \pm 3.70$ & $21.84 \pm 0.58^{*}$ & $20.48 \pm 0.87^{*}$ \\
7 & $\mathrm{CIP}$ & $14.45 \pm 1.4$ & $14.74 \pm 0.67^{*}$ & $14.21 \pm 0.47^{*}$ \\
8 & $\mathrm{MA}$ & $525.45 \pm 60.50$ & $605.36 \pm 29.47^{*}$ & $529.49 \pm 31.65^{*}$
\end{tabular}

Note: differences are significant in comparison with the healthy group, ${ }^{*}-p<0.05$

In the second group, statistically significant $(\mathrm{p}<0.05)$ normalization of all indicators characterizing hemocoagulation potential of blood CTA, BCT, ICD, CIP, MA and indicators A0, R (t1) and ICCcharacterizing platelet aggregation capacity, as well as an indicator responsible for changes in the fibrinolytic blood system. The recorded amplitude of the indicator of the intensity of the contact phase coagulation (ICC) $84.41 \pm 1.61$ r.u. (in the 2 nd group), at the rate of $84.3 \pm 10.91$ r.u., the initial rate of aggregation of blood at the time of A0 $220.43 \pm 15.61$ r.u. (in the 2 nd group), at the rate of $222.25 \pm 15.33$ r.u., the coagulation contact phase time $\mathrm{R}(\mathrm{t} 1)$ is $2.37 \pm 0.15$ minutes (in the 2 nd group) at the rate of $2.36 \pm 0.34$, all of which indicates a reliable normalization of these parameters. Normalization of the amplitude and time constants of hemocoagulation is also noted. The nature of the thromboelastogram indicates the normalization of all hemostatic system on the background of treatment on the 7th day after surgery.

Fig. 3 shows the indicators of LPTEG on day 7 in both groups and in healthy volunteers. Fig. 4 shows the dynamics of A0 LPTEG in both groups. Fig. 5 shows the dynamics of ICC LPTEG in both groups. Fig. $\mathbf{6}$ shows the dynamics of ICD LPTEG in both groups. Fig. 7 shows the dynamics of MA LPTEG in both groups.

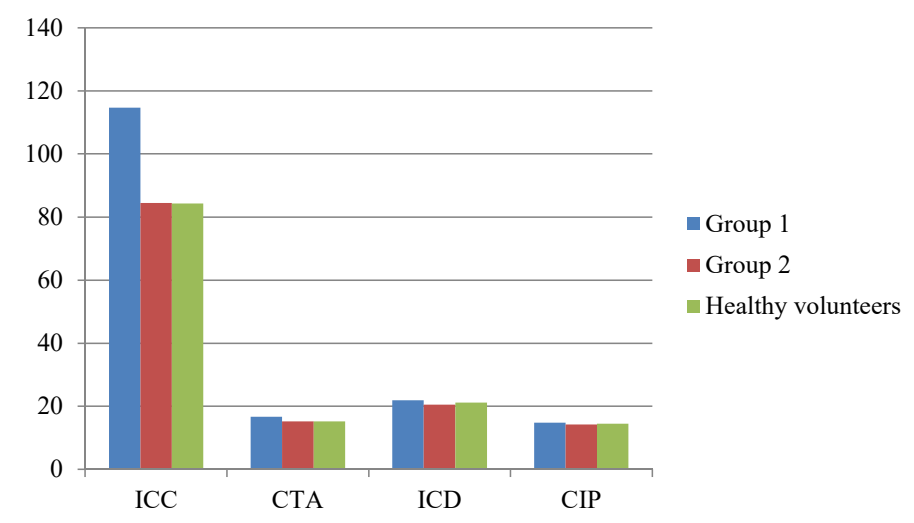

Fig. 3. Indicators of LPTEG on day 7 in both groups and in healthy volunteers 


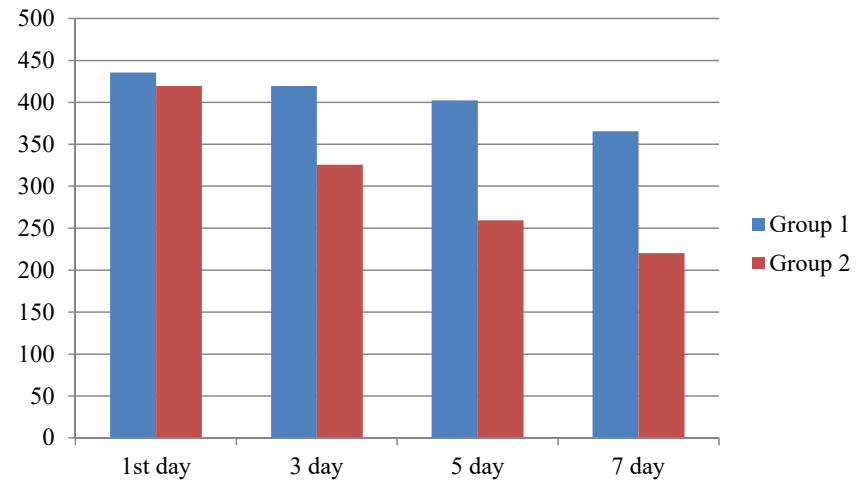

Fig. 4. Dynamics of A0 LPTEG in both groups

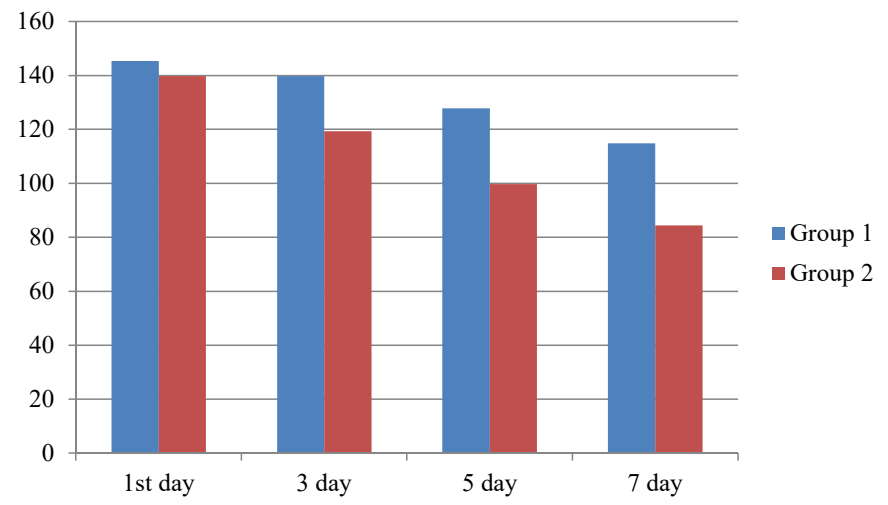

Fig. 5. Dynamics of ICC LPTEG in both groups

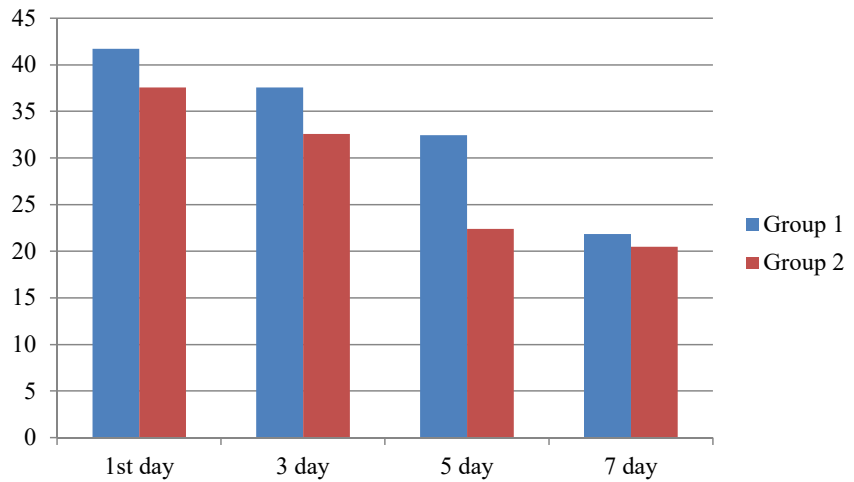

Fig. 6. Dynamics of ICD LPTEG in both groups

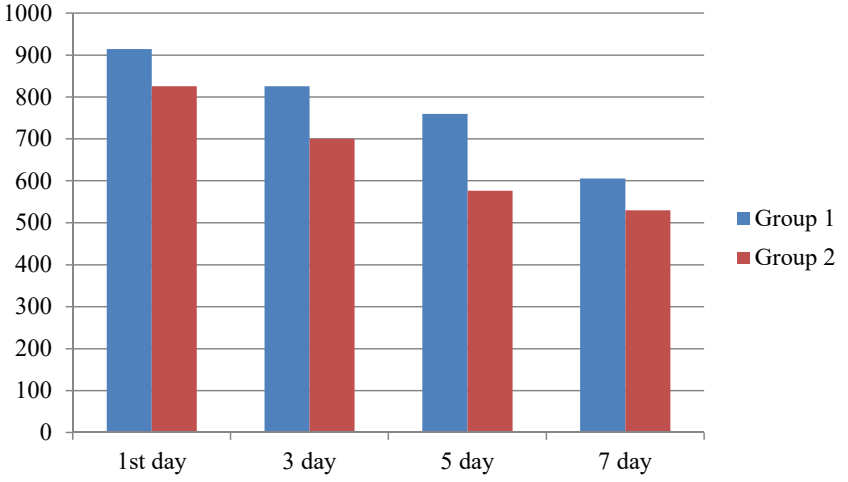

Fig. 7. Dynamics of MA LPTEG in both groups 


\section{Discussion}

Analysis of the hemostasis system in patients with OA using NPTEG in dynamics shows the possibility of using the NPTEG method for early diagnosis of the degree of hemostasis disorders and control of anticoagulant therapy.

From the above figures and tables, we can see that the differential correction of hemostatic system disorders, namely the use of rivaroxoban in the postoperative period and the NTPEG method, contributed to the improvement of the coagulation and fibrinolytic units of the hemostasis system at all stages of surgical treatment. Rivaroxaban as a new anticoagulant is now gaining popularity thanks to a safer use profile.

The effect of rivaroxaban on different categories of patients and in combination with different drugs is now widely studied.

Comparing our data with the COMPASS study [23], we can state the effectiveness of the use of rivaroxoban for the treatment and prevention of thromboembolic events in this category of patients. According to the COMPASS study, the success of rivaroxban for the prevention of secondary cardiovascular events is shown. The final results show that the combination of rivaroxaban plus aspirin was associated with a significantly smaller number of events of the primary outcome, compared with taking aspirin alone, including patients with obliterating atherosclerosis.

Continuing this study, it is possible to study the feasibility of prolonged use of rivaroxaban in this category of patients with the goal of preventing retrombosis in the area of shunt.

Limitations of the study: the limitations of this study is the impossibility of conducting a study in patients previously taking anticoagulants for concomitant pathology.

In the future prospect of further research, we hope to show the most rational scheme for the prevention of thromboembolic events in this category of patients. Also, we want to demonstrate the possibilities of using LFTEG to monitor the state of the hemostatic system during treatment and in order to further control the dosage.

\section{Conclusions}

Significant structural and chronometric hypercoagulation with increased thrombin generation and inhibition of fibrinolytic activity of blood was noted before surgery and the beginning of treatment in patients with OA on the background of activation of the vascular-platelet hemostasis.

Against the background of treatment in group 1, the complex of the epidural administration of bupivacaine, enoxaparin $0.4 \mathrm{ml}$ (40,000 anti-HA MO) 2 times a day from the first postoperative day and pentoxifylline IV $200 \mathrm{mg}$ twice a day were observed reliable changes toward normalization in all components of the hemostasis system according to the NTPEG method $(\mathrm{p}<0.001)$. The fibrinolytic system in group 1 was suppressed at day 7 of treatment $(p<0.001)$ compared to normal but has positive dynamics compared to day $1(\mathrm{p}<0.001)$. Platelet aggregation is increased compared to the norm $(\mathrm{p}<0.001)$ and has significant $(\mathrm{p}<0.001)$ differences compared with the indicators before treatment in group 1. Intensive care of group 1 on the background of systemic pathological process led to normalization of the coagulation link of the hemostasis system in patients with OA. Vascular-platelet and fibrinolytic systems did not reach normal values in 7 days, but showed a significant tendency to decrease the degree of manifestations of their disorders $(p<0.001)$.

On the background of treatment of the 2 nd group in the composition of the epidural administration of bupivacaine, rivaroxaban $15 \mathrm{mg} 2$ times daily orally from the first postoperative day, significant changes towards normalization were observed in all components of the hemostasis system by the method of LPTEG $(\mathrm{p}<0.001)$. The state of the coagulation unit of the hemostasis system in patients with OA for 7 days of therapy does not differ significantly from the indicators of the norm, it is possible to note significant shifts to hypocoagulation within the norm $(\mathrm{p}<0.05)$. The fibrinolytic system at the 7th day of treatment is not statistically different from the norms, has a positive dynamics compared to 1 day $(\mathrm{p}<0.001)$. Platelet aggregation is also not statistically different from normal values and has significant differences compared with rates of 5 and 1 day $(\mathrm{p}<0.05 ; \mathrm{p}<0.001)$.

In the second group, statistically significant $(\mathrm{p}<0.05)$ normalization of all indicators that characterize the hemocoagulation potential of blood CTA, BCT, CIP, ICD, MA and indicators A0, 
$\mathrm{R}$ (t1) and ICC, which characterize the aggregation capacity of platelets, were found. as well as the indicator responsible for changes in the fibrinolytic blood system.

\section{Conflict of interest}

No conflict of interest.

\section{References}

[1] Hess, C. N., Norgren, L., Ansel, G. M., Capell, W. H., Fletcher, J. P., Fowkes, F. G. R. et. al. (2017). A Structured Review of Antithrombotic Therapy in Peripheral Artery Disease With a Focus on Revascularization. Circulation, 135 (25), $2534-2555$. doi: http://doi.org/10.1161/circulationaha.117.024469

[2] Zannad, F., Bauersachs, R. (2018). Rivaroxaban: A New Treatment Paradigm in the Setting of Vascular Protection? Thrombosis and Haemostasis, 118 (S 01), S12-S22. doi: http://doi.org/10.1055/s-0038-1636530

[3] Anand, S. (2017). On Behalf of COMPASS Investigators. Rivaroxaban in stable peripheral or carotid artery disease. Presented at the European Society of Cardiology Congress 2017. Barcelona.

[4] Harwood, A.-E., Smith, G. E., Cayton, T., Broadbent, E., Chetter, I. C. (2016). A Systematic Review of the Uptake and Adherence Rates to Supervised Exercise Programs in Patients with Intermittent Claudication. Annals of Vascular Surgery, 34, 280-289. doi: http://doi.org/10.1016/j.avsg.2016.02.009

[5] Layden, J., Michaels, J., Bermingham, S., Higgins, B. (2012). Diagnosis and management of lower limb peripheral arterial disease: summary of NICE guidance. BMJ, 345 (1), e4947. doi: http://doi.org/10.1136/bmj.e4947

[6] Creager, M. A., Libby, P. (2012). Peripheral Artery Diseases. Braunwald's Heart Disease: A Textbook of Cardiovascular Medicine. Philadelphia: Elsevier 1338-1358. doi: http://doi.org/10.1016/b978-1-4377-0398-6.00061-5

[7] Secemsky, E. A., Schermerhorn, M., Carroll, B. J., Kennedy, K. F., Shen, C., Valsdottir, L. R. et. al. (2017). Readmissions After Revascularization Procedures for Peripheral Arterial Disease. Annals of Internal Medicine, 168 (2), 93-99. doi: http:// doi.org/10.7326/m17-1058

[8] Gavrilenko, A. V., Al-Yousef, N. N., Wang, X., Mamedova, N. M., Ananieva, M. V., Li, R. (2019). Profundoplasty in treatment of patients with chronic lower limb ischaemia. Angiology and Vascular Surgery, 25 (3), 122-127. doi: http://doi.org/10.33529/ angio2019308

[9] Fowkes, F. G. R., Rudan, D., Rudan, I., Aboyans, V., Denenberg, J. O., McDermott, M. M. et. al. (2013). Comparison of global estimates of prevalence and risk factors for peripheral artery disease in 2000 and 2010: a systematic review and analysis. The Lancet, 382 (9901), 1329-1340. doi: http://doi.org/10.1016/s0140-6736(13)61249-0

[10] Eikelboom, J. W., Connolly, S. J., Bosch, J., Dagenais, G. R., Hart, R. G., Shestakovska, O. et. al. (2017). Rivaroxaban with or without Aspirin in Stable Cardiovascular Disease. New England Journal of Medicine, 377 (14), 1319-1330. doi: http:// doi.org/10.1056/nejmoa1709118

[11] Giatti, L., Camelo, L. do V., Rodrigues, J. F. de C., Barreto, S. M. (2012). Reliability of the MacArthur scale of subjective social status - Brazilian Longitudinal Study of Adult Health (ELSA-Brasil). BMC Public Health, 12 (1). doi: http:// doi.org/10.1186/1471-2458-12-1096

[12] Rooke, T. W., Hirsch, A. T., Misra, S., Sidawy, A. N., Beckman, J. A., Findeiss, L. K. et. al. (2011). 2011 ACCF/AHA Focused Update of the Guideline for the Management of Patients With Peripheral Artery Disease (Updating the 2005 Guideline). Journal of the American College of Cardiology, 58 (19), 2020-2045. doi: http://doi.org/10.1016/j.jacc.2011.08.023

[13] Prévost, A., Lafitte, M., Pucheu, Y., Couffinhal, T. (2013). Education and home based training for intermittent claudication: functional effects and quality of life. European Journal of Preventive Cardiology, 22 (3), 373-379. doi: http:// doi.org/10.1177/2047487313512217

[14] McDermott, M. M. (2013). Functional Impairment in Peripheral Artery Disease and How to Improve It in 2013. Current Cardiology Reports, 15 (4). doi: http://doi.org/10.1007/s11886-013-0347-5

[15] Kullo, I. J., Rooke, T. W. (2016). Peripheral Artery Disease. New England Journal of Medicine, 374 (9), 861-871. doi: http:// doi.org/10.1056/nejmcp1507631

[16] Kearon, C., Akl, E. A., Comerota, A. J., Prandoni, P., Bounameaux, H., Goldhaber, S. Z. et. al. (2012). Antithrombotic Therapy for VTE Disease. Chest, 141 (2), e419S-e496S. doi: http://doi.org/10.1378/chest.11-2301

[17] Connolly, S. J., Eikelboom, J. W., Bosch, J., Dagenais, G., Dyal, L., Lanas, F. et. al. (2018). Rivaroxaban with or without aspirin in patients with stable coronary artery disease: an international, randomised, double-blind, placebo-controlled trial. The Lancet, 391 (10117), 205-218. doi: http://doi.org/10.1016/s0140-6736(17)32458-3

[18] Tarabrin, O., Shcherbakov, S., Gavrychenko, D., Saleh, O., Lyoshenko, I., Kushnir, O. (2013). How to control the coagulation disorders? European Journal of Anaesthesiology, 30, 92-92. doi: http://doi.org/10.1097/00003643-201306001-00285 
[19] Tagalakis, V., Patenaude, V., Kahn, S. R., Suissa, S. (2013). Incidence of and Mortality from Venous Thromboembolism in a Real-world Population: The Q-VTE Study Cohort. The American Journal of Medicine, 126 (9), 13-21. doi: http://doi.org/ 10.1016/j.amjmed.2013.02.024

[20] Sapelkin, S. V., Kuznetsov, M. R. (2016). Konservativnoye lecheniye bol'nykh s zabolevaniyami perifericheskikh arteriy: vozmozhnosti i sushchestvuyushchiye problemy. Angiologiya i sosudistaya khirurgiya, 4, 169-175.

[21] Ferreira, V., Freixo, C., Gonçalves, J., Teixeira, G., Antunes, I., Veiga, C. et. al. (2019). Effectiveness and Safety of Rivaroxaban Compared to Acenocumarol after Infrainguinal Surgical Revascularization. Annals of Vascular Surgery, 55, 78-84. doi: http:// doi.org/10.1016/j.avsg.2018.06.008

[22] Halle, T. R., Benarroch-Gampel, J., Teodorescu, V. J., Rajani, R. R. (2018). Surgical Intervention for Peripheral Artery Disease Does Not Improve Patient Compliance with Recommended Medical Therapy. Annals of Vascular Surgery, 46, $104-111$. doi: http://doi.org/10.1016/j.avsg.2017.06.152

[23] Bhagirath, V. C., Eikelboom, J. W., Anand, S. S. (2018). Low-dose rivaroxaban plus aspirin for the prevention of cardiovascular events: an evaluation of COMPASS. Future Cardiology, 14 (6), 443-453. doi: http://doi.org/10.2217/fca-2018-0059

\title{
MAGNETIC RESONANCE IMAGING WITH DIFFUSE WEIGHTED IMAGING AND COMPUTED TOMOGRAPHY WITH INTRAVENOUS CONTRAST IN STAGING OF DISSEMINATED OVARIAN, STOMACH, COLORECTAL CANCER
}

\author{
Sergiy Chetverikov \\ Surgery department No.4 with oncology course ${ }^{l}$ \\ chetverikov@rambler.ru \\ Svitlana Zavoloka \\ Surgery department No. 4 with oncology course \\ zavsvetlana.od@gmail.com \\ Viacheslav Onyshchenko \\ Department of simulation medicine \\ Odessa National Medical University \\ 2 Valichovskiy lane, Odessa, Ukraine, 65082 \\ vicedeanodessa@gmail.com \\ Mykhailo Chetverikov ${ }^{1}$ \\ chetvericov@yahoo.com \\ Valeriia Chetverikova-Ovchynnyk ${ }^{l}$ \\ ovchinnik.val@yahoo.com \\ ${ }^{1}$ Center of Reconstructive and Restorative Medicine \\ University Clinic of Odessa National Medical University \\ 8 Tenista str., Odessa, Ukraine, 65009
}

Abstract

The aim of the research. Development and implementation of new methods for pre-operative staging of advanced ovarian, gastric and colorectal cancer to improve patient selection for cytoreductive surgery and increase its radicality. 